\title{
Effets des embruns marins pollués sur les feuilles d'Acacia cyanophylla Lindl. et d'Eucalyptus gomphocephala Dc. du littoral tunisien
}

\author{
Naceur El Ayeb ${ }^{a *}$, Belgacem HenCHI ${ }^{b}$, Jean Pierre GARREC ${ }^{c}$, Mohamed Néjib ReJEB $^{d}$ \\ ${ }^{a}$ Institut Supérieur de Biotechnologie de Monastir, BP 10, 5000 Monastir, Tunisie \\ ${ }^{\mathrm{b}}$ Faculté des Sciences de Tunis, 1060 Tunis, Tunisie \\ ${ }^{c}$ Centre des Recherches Forestières, INRA, Champenoux, 54280 Nancy, France \\ ${ }^{\mathrm{d}}$ Institut National des Recherches en Génie Rural, Eaux et Forêts, BP 2, 2080 Ariana, Tunisie
}

(Reçu le 5 août 2002 ; accepté le 9 avril 2003)

\begin{abstract}
Résumé - Un dépérissement de la végétation côtière du Cap Bon en Tunisie est observable depuis ces deux dernières décennies. En de nombreux points de la côte, les peuplements d'Acacia cyanophylla Lindl. et d'Eucalyptus gomphocephala Dc. sont touchés. Les feuilles des arbres dépérissants présentent une réduction marquée de leur teneur relative en eau par rapport à celle des feuilles saines, avec des altérations de leur surface au niveau des cires épicuticulaires. Ces dépérissements sont liés à la présence récente dans les embruns marins de plusieurs polluants comme des tensioactifs anioniques. Les résultats d'expériences en laboratoire confirment l'hypothèse que lorsque les embruns marins contiennent des tensioactifs et des hydrocarbures, ils ont alors un impact très important sur les feuilles avec en particulier une altération de la cuticule. En effet les ions $\mathrm{Na}^{+}, \mathrm{Cl}^{-}$et les polluants présents dans les embruns peuvent alors franchir largement la barrière cuticulaire et exercer leurs effets néfastes sur les tissus foliaires.
\end{abstract}

dépérissement forestier / embruns marins pollués / alkylebenzène sulfonates linéaire / cire cuticulaire

Abstract - Effects of polluted sea-spray on leaves of Acacia cyanophylla Lindl. and Eucalyptus gomphocephala Dc. in Tunisian coast. A deterioration of the coastal vegetation of the Cape Bon in Tunisia has been observable for these two last decades. In many points of the coast, the peuplement of Acacia cyanophylla Lindl. and Eucalyptus gomphocephala Dc. are touched. The sheets of the damaged trees present a marked reduction of their relative content water compared to that of the healthy sheets, with deterioration of their surface on the epicuticular waxes. These deterioration are related to the recent presence in the marine spray of several pollutants like anionic surfactants. The experiences results in laboratory confirm hypothesis that when the sea-spray contains the surfactant and hydrocarbons, they then have a very significant impact on the sheets with in particular a deterioration of the cuticle. Indeed $\mathrm{Na}^{+}$and $\mathrm{Cl}^{-}$ions and the pollutants present in the spray can then largely cross the cuticular barrier and exert their deleterious effects on leaf tissues.

forest decline / polluted sea-spray / linear alkylbenzene sulfonates / cuticular wax

\section{INTRODUCTION}

Depuis les années 1960, des dépérissements de la végétation littorale sont constatés en de nombreux points de la côte méditerranéenne [37]. En Tunisie, ce phénomène présente depuis le début des années 1980 une évolution préoccupante [12]. Il a été mis en évidence sur le littoral du Cap Bon un dépérissement des arbres forestiers, essentiellement sur l'Acacia cyanophylla et l'Eucalyptus gomphocephala, semblable à celui observé en Italie sur les pinèdes (Pinus pinea, Pinus pinaster) du parc de San Rossore situé sur la côte tyrrhénienne [20] et aussi à celui observé en France sur les pins d'Alep de la côte méditerranéenne [10]. Ce dépérissement forestier observé sur les côtes de France et d'Italie résulte de la présence «récente » dans les embruns d'un ou plusieurs composés phytotoxiques [17]. Les tensioactifs anioniques présents dans les détergents apportés par les eaux usées des agglomérations du littoral, et les hydrocarbures rejetés par le trafic maritime sont au premier rang des substances impliquées [36]. Comme le littoral tunisien s'ouvre uniquement sur la mer Méditerranéenne, il y'a de fortes chances d'une origine commune de ces différents dépérissements côtiers méditerranéens [14]. Pour vérifier cette hypothèse, il a été dosé : d'une part les polluants de type tensioactifs anioniques non biodégradables présents dans la couche superficielle de la mer le long des côtes tunisiennes, et d'autre part les mêmes polluants sur les feuilles des arbres du littoral. Toujours dans le cadre de cette hypothèse, à partir d'expériences de simulation en laboratoire, il a été cherché à reproduire les symptômes caractéristiques du dépérissement observé in situ. Parallèlement, pour comprendre les mécanismes physiologiques à l'origine de

\footnotetext{
* Corresponding author: Naceur.ElAyeb@isbm.rnu.tn
} 
ces dépérissements, les statuts hydrique et minéral des feuilles sous l'effet des embruns marins pollués ont été particulièrement étudiés.

\section{MATÉRIELS ET MÉTHODES}

\subsection{Matériel végétal}

In situ des prélèvements foliaires ont été effectués sur un peuplement d'acacia (Acacia cyanophylla Lindl.) et d'eucalyptus (Eucalyptus gomphocephala Dc.) de la forêt domaniale Menzel Belgacem du Cap Bon située en bordure de la mer (stations de Oued El Bir et de Oued El Ksab). Les mêmes espèces ont été retenues pour les expérimentations au laboratoire. Elles sont largement utilisées pour le reboisement des régions littorales des zones subhumides de Tunisie [1].

\subsection{Méthodes}

\subsection{1. Études effectuées in situ}

\subsubsection{Paramètres hydriques des feuilles}

Des prélèvements de feuilles ont été effectués sur 10 arbres d'A. cyanophylla et sur 10 arbres d'E. gomphocephala: 20 feuilles nécrosées ont été prélevées sur des arbres dépérissants et 20 feuilles témoins ont été prélevées sur des arbres sains. La teneur relative en eau (RWC) a été déterminée sur ces feuilles à partir de la relation : $\mathrm{RWC}=(\mathrm{MF}-\mathrm{MS}) /(\mathrm{MFsat}-\mathrm{MS}) \times 100$ avec $\mathrm{MF}$ : poids de la matière végétale fraîche ; MS: poids de la matière végétale sèche ; MFsat : poids de la matière végétale à saturation en eau.

\subsubsection{Paramètres minéraux des feuilles}

L'analyse des teneurs en macroéléments $\mathrm{Na}^{+}$et $\mathrm{K}^{+}$des différentes feuilles prélevées est réalisée par spectrophotométrie de flamme (appareil Jenway $\mathrm{PFP}_{7}$ ) sur les solutions acides de cendres obtenues après calcination des feuilles réduites en poudre. De son côté le $\mathrm{Ca}^{++}$ est dosé dans la même solution par titrage direct à l'aide du versenate en présence d'un indicateur coloré, le murexide, en milieu convenablement tamponné ( $\mathrm{pH}=12$ ).

\subsubsection{Mouillabilité des surfaces foliaires}

La mouillabilité est estimée à partir de la mesure de l'angle de contact de gouttes d'eau $\left(\theta^{\circ}\right)$ déposées sur la surface foliaire. Comme cette mouillabilité dépend directement des caractéristiques de la surface foliaire, cet angle nous renseignera donc également sur l'état des cires épicuticulaires : qualité et quantité. L'angle de contact et la mouillabilité sont deux paramètres qui évoluent en sens inverse : plus l'angle de contact est faible, plus la mouillabilité est grande. Les mesures ont été effectuées sur des feuilles fraîches d'acacia et d'eucalyptus. Le volume de la goutte d'eau déposée au moyen d'une micro-pipette est de $0,5 \mu \mathrm{L}$ et l'observation est réalisée sous un microscope optique avec un grossissement de $50 \times$. Il a été procédé à 5 répétitions par feuille en utilisant 10 feuilles par plante.

\subsubsection{Dosage des tensioactifs anioniques (LAS)}

Une méthode titrimétrique utilisant une électrode spécifique (High Sens Tenside Electrode, Orion) a été utilisée pour le dosage des LAS (Linear alkylbenzene sulfonates: composés actifs des détergents) directement dans les échantillons d'eau de mer (sur une couche d'environ $2 \mathrm{~mm}$ d'épaisseur). L'étalonnage de cette électrode est effectué au moyen d'un tensioactif cationique : le 1,3 didecyl-2-methyl-imidazolechlorure (D.D.M.I.C.I.). De son côté le dosage des LAS présents sur les surfaces foliaires s'effectue après un trempage rapide $(25 \mathrm{~s})$ des feuilles d'acacia et d'eucalyptus dans du chloroforme ( $0,5 \mathrm{~g}$ de MF de feuille pour $10 \mathrm{~mL}$ de chloroforme), ceci de façon à séparer les LAS fixés par leurs pôles lipophiles aux lipides des cires cuticulaires sans pour autant extraire ces cires. Après l'extraction, le chloroforme est évaporé et les LAS sont mis en solution dans de l'eau distillée avant dosage au moyen d'une électrode spécifique.

\subsection{2. Études effectuées au laboratoire}

\subsubsection{Expériences de simulation}

Les expériences sont réalisées au moyen d'un brumisateur mobile générant des aérosols salins de différentes tailles. Pour éviter l'infiltration des embruns dans le sol, un disque en plastique est placé au niveau du collet pour chaque plant. Cette étude a concerné des jeunes plants d'A. cyanophylla et d'E. gomphocephala âgés de 6 mois élevés en serre ( 300 plants soit 2 espèces $\times 5$ traitements $\times 30$ plants). Les plants ont été exposés à différents brouillards salins plus ou moins enrichis en polluants : LAS et hydrocarbures (HC). Le tensioactif anionique testé a été le $\mathrm{C}_{12} \mathrm{H}_{25}$-benzène- $\mathrm{SO}_{3} \mathrm{Na}$ et l'hydrocarbure retenu est une fraction pétrolière distillée semblable à celle qu'on retrouve sur la surface de la mer. Cinq traitements différents ont été appliqués sur la partie aérienne des jeunes plants : eau distillée (ED) : témoin ; eau de mer synthétique (EMS) : environ $38 \mathrm{~g}$ de sels/L ; eau de mer synthétique $+50 \mu \mathrm{g} / \mathrm{L}$ de tensioactifs anioniques (EMS + LAS); eau de mer synthétique $+50 \mu \mathrm{g} / \mathrm{L}$ de tensioactifs anioniques $+1,25 \mathrm{~mL}$ de fuel/ litre d'eau de mer (EMS + LAS + HC) ; eau de mer polluée naturelle (EMP) : prélevée in situ au Cap Bon.

Le choix de la concentration en LAS a été fixé à partir des concentrations connues en tensioactifs anioniques à la surface de l' eau de mer [12]. L'eau de mer synthétique [34] est constituée de (q.s.p. 10 L) : $\mathrm{CaCl}_{2}, 6 \mathrm{H}_{2} \mathrm{O}=11,0442 \mathrm{~g} ; \mathrm{NaHCO}_{3}=1,9278 \mathrm{~g} ; \mathrm{KBr}=0,96 \mathrm{~g} ; \mathrm{H}_{3} \mathrm{BO}_{3}=$ $0,2724 \mathrm{~g} ; \mathrm{KCl}=6,6618 \mathrm{~g} ; \mathrm{Na}_{2} \mathrm{SO}_{4}=39,2 \mathrm{~g} ; \mathrm{MgCl}_{2}, 6 \mathrm{H}_{2} \mathrm{O}=106,43 \mathrm{~g}$; $\mathrm{NaCl}=234$ g et $\mathrm{SrCl}_{2}, 6 \mathrm{H}_{2} \mathrm{O}=0,4$ g. Pendant 60 jours, les plants ont été exposés une fois par jour au laboratoire aux embruns salins pollués jusqu'au point de goutte des feuilles (environ 20 min d'exposition par traitement).

Pour classer les altérations apparaissant sur les feuilles suite aux différents traitements, 6 niveaux de dommage sont définis en fonction du \% de surface foliaire nécrosée $\mathrm{S} ; \mathrm{n}_{0}: \mathrm{S}<5 \% ; \mathrm{n}_{1}: 5 \leq \mathrm{S}<20 \% ; \mathrm{n}_{2}$ : $20 \leq \mathrm{S}<40 \% ; \mathrm{n}_{3}: 40 \leq \mathrm{S}<60 \% ; \mathrm{n}_{4}: 60 \leq \mathrm{S}<80 \% ; \mathrm{n}_{5}: \mathrm{S}>80 \%$.

\subsubsection{Observations au microscope électronique à balayage}

Les cires épicuticulaires présentes sur les surfaces foliaires ont été examinées en microscopie électronique à balayage (appareil Streoscan 90B, Cambridge Instruments, UK) sous une tension accélératrice de $15 \mathrm{KV}$ après métallisation au carbone des feuilles. Pour quantifier les modifications de l'état de surface des feuilles au niveau des cires épicuticulaires, nous avons utilisé les critères de classement des dommages de Sauter et Voss [33] :

- État I : pas de dégradation ni d'agrégation des cires épicuticulaires ou épistomatiques.

- État II : début de dégradation et d'agglomération des cires épicuticulaires (disparition des structures cireuses inférieure à $30 \%$ ) et début d'agrégation des cires épistomatiques (sur au moins $1 / 3$ de la surface des stomates).

- État III : dégradation et disparition des cires épicuticulaires de 30 à $80 \%$. Agrégation qui touche une moyenne ou forte proportion des cires épistomatiques (1/3 à 8/10 de la surface des stomates).

- État IV : disparition presque totale des cires épicuticulaires. Agrégation très importante des cires épistomatiques (formation d'une couche continue de cire sur presque 9/10 de la surface des stomates). 


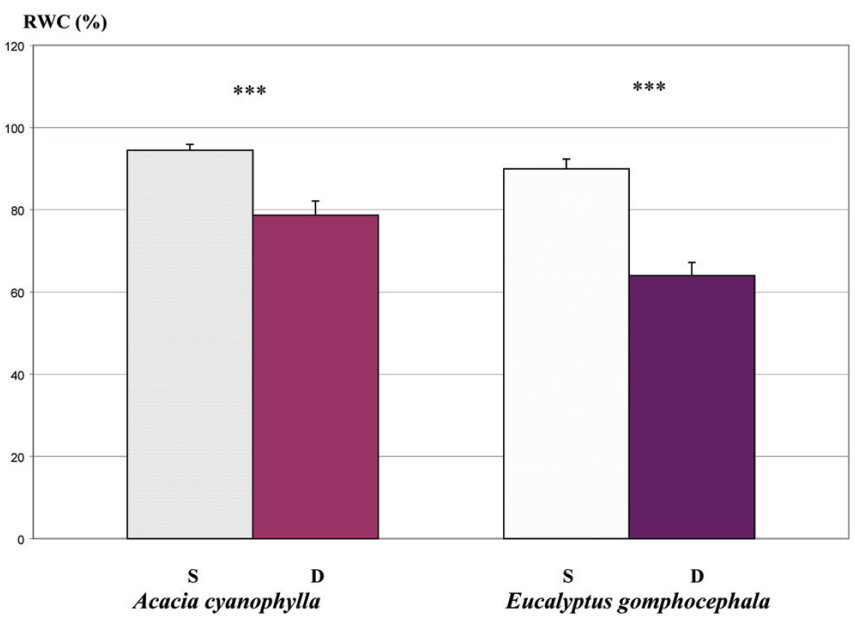

Figure 1. Variation de la teneur relative en eau (RWC en \%) de feuilles d'Acacia cyanophylla et d'Eucalyptus ghomphocephala sains (S) et dépérissants (D) $(n=49)$. Variation très hautement significative $(p<0,001: * * *)$. Les barres verticales correspondent à l'écart type.

Sur tous les arbres, la quantification des nécroses foliaires, l'étude des altérations des surfaces foliaires et de la mouillabilité ont été effectuées 30 jours après les dernières fumigations.

\subsubsection{Exploitation statistique}

Sur les différents résultats obtenus nous avons effectué une analyse de la variance (Anova) basée sur le test de Fisher (au risque $\alpha=5 \%$ ). Les valeurs étaient significativement différentes avec : variation significative $(p<0,05: *)$, hautement significative $(p<0,01: * *)$ et très hautement significative $(p<0,001: * * *)$.

\section{RÉSULTATS}

\subsection{Effets des embruns marins pollués sur les arbres in situ}

\subsection{1. Évolution des paramètres hydriques}

En comparant la teneur relative en eau des feuilles nécrosées par rapport aux feuilles saines, nous mettons en évidence que le dépérissement résulte d'une nette baisse de la teneur relative en eau (RWC), celle-ci étant plus ou moins marquée selon l'espèce : $29 \%$ chez E. gomphocephala et seulement $17 \%$ chez A. cyanophylla (Fig. 1).

\subsubsection{Analyse minérale foliaire}

Nous constatons que la teneur en $\mathrm{Na}^{+}$foliaire $(\%$ du poids de MS) d'A. cyanophylla augmente de 0,54 à 1,62 lorsque l'on se rapproche de la ligne de rivage. $\mathrm{Si}$ ce gradient de concentration est croissant pour $\mathrm{Na}^{+}$, il est au contraire décroissant pour $\mathrm{Ca}^{++}$(Fig. 2). De même chez, E. gomphocephala, on observe que la teneur en $\mathrm{Na}^{+}$augmente fortement lorsque l'on se rapproche de la côte, alors qu' au contraire la concentration foliaire en $\mathrm{K}^{+}$ diminue nettement (Fig. 2).
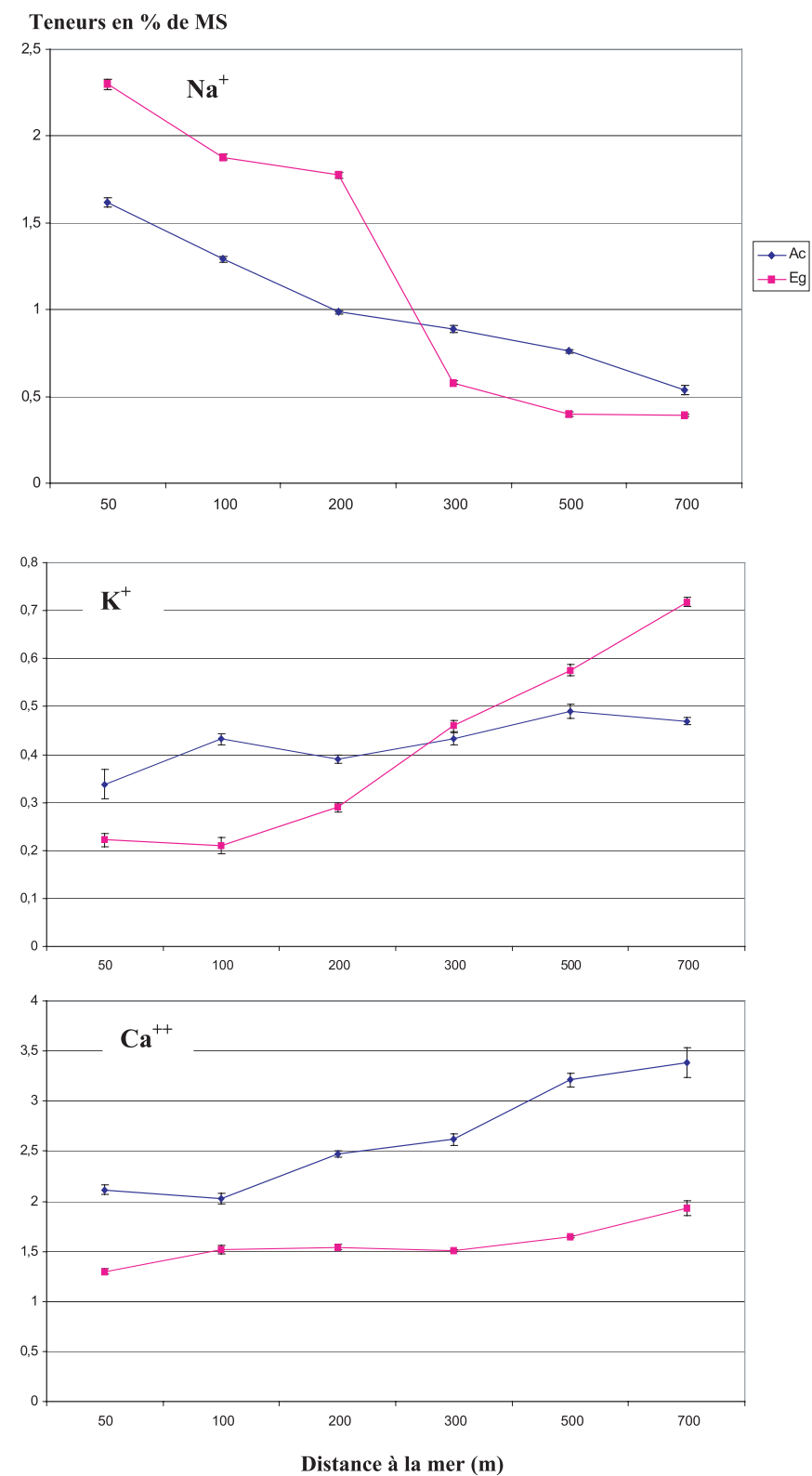

Figure 2. Variation des teneurs foliaires en $\mathrm{Na}^{+}, \mathrm{K}^{+}$et $\mathrm{Ca}^{++}$(exprimées en \% de MS) d'Acacia cyanophylla (Ac) et d'Eucalyptus gomphocephala $(\mathrm{Eg})$ in situ en fonction de l'éloignement de la ligne de rivage. Les barres verticales correspondent à l'écart type.

\subsubsection{Mouillabilité des surfaces}

L'étude de l'angle de contact des gouttes d'eau sur des feuilles saines et nécrosées prélevées in situ fait apparaître que le dépérissement se caractérise par une diminution de cet angle $\theta^{\circ}$ avec pour conséquence une augmentation de la mouillabilité des feuilles. Chez A. cyanophylla, cet angle diminue de $26 \%$ pour les feuilles nécrosées par rapport aux feuilles saines, et comparativement cette diminution est de $29 \%$ chez E. gomphocephala (Fig. 3). 
Tableau I. Concentrations des LAS (exprimée $\mu \mathrm{g} / \mathrm{L}$ ) dans la couche supérieure de l'eau de mer (Station O. El Ksab).

\begin{tabular}{lccccccccccccc}
\hline $\mathbf{N}^{\circ}$ des mesures & 1 & 2 & 3 & 4 & 5 & 6 & 7 & 8 & 9 & 10 & 11 & 12 & Moy. \\
\hline Eau de mer & 6,9 & 12,2 & 8,9 & 7,9 & 0 & 13,3 & 16,5 & 15,0 & 13,8 & 12,5 & 12,6 & 10,0 & 10,8 \\
\hline
\end{tabular}

Tableau II. Concentrations des LAS (exprimée en $\mu \mathrm{g} / \mathrm{kg}$ de MF) présents sur les feuilles d'E. gomphocephala (Station O. El Ksab).

\begin{tabular}{lccccccccccc}
\hline $\mathbf{N}^{\circ}$ des mesures & 1 & 2 & 3 & 4 & 5 & 6 & 7 & 8 & 9 & 10 & Moy. \\
\hline Eucalyptus & 13,6 & 17,6 & 12 & 13,8 & 13,5 & 11,9 & 13,8 & 15,6 & 12,2 & 14,1 & 13,8 \\
\hline
\end{tabular}

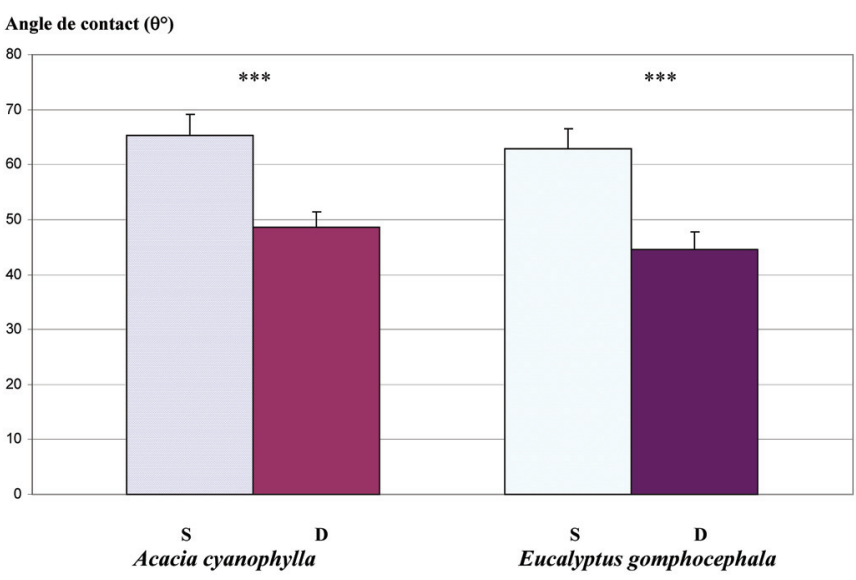

Figure 3. Variation de l'angle de contact des gouttes d'eau $\left(\theta^{\circ}\right)$ sur les feuilles d'Acacia cyanophylla et d'Eucalyptus gomphocephala sains (S) et dépérissants (D) $(n=260)$. Variation très hautement significative $(p<0001: * * *)$. Les barres verticales correspondent à l'écart type.

\subsubsection{Dosage des LAS dans l'eau de mer et sur les feuilles in situ}

Pour ces premières mesures réalisées dans cette région, nous avons trouvé des concentrations de 10,8 $\pm 0,1 \mu \mathrm{g} / \mathrm{L}$ de tensioactifs anioniques dans la couche superficielle d'eau de mer de la station de Oued El Ksab (Tab. I). Parallèlement nous avons déterminé des concentrations de LAS de 13,8 $\pm 0,1 \mu \mathrm{g} / \mathrm{kg}$ de MF sur les feuilles d'E. gomphocephala exposés directement aux embruns marins pollués dans la même station (Tab. II).

\subsection{Effets des embruns marins pollués sur les arbres au laboratoire}

\subsubsection{Simulation du phénomène de dépérissement}

L'étude des niveaux de dommage sur les feuilles d'A. cyanophylla et d'E. gomphocephala sains ou ayant subi les différents traitements polluants met en évidence que les plants témoins (ED) ne montrent aucune nécrose $\left(100 \% \mathrm{n}_{0}\right)$ alors que les traitements EMS, EMS + LAS et EMS + LAS + HC entraînent de nettes nécroses foliaires sur les deux espèces d'arbres (Fig. 4). Si nous constatons que l'eau de mer synthétique (traitement EMS) est déjà à l'origine de nécroses foliaires chez l'eucalyptus, en comparant les effets des traitements EMS et
EMS + LAS, on peut estimer en prenant le pourcentage de feuilles fortement nécrosées (niveau $\mathrm{n}_{5}$ ), que la présence des LAS entraîne une augmentation de l'impact du sel de 7 à $30 \%$ (sans pouvoir exclure également un impact propre aux LAS) (Fig. 4). De même, en comparant les traitements EMS + LAS et EMS + LAS + HC, on peut estimer comme précédemment en prenant les \% de feuilles nécrosées au niveau $\mathrm{n}_{5}$, que la présence des HC en plus des LAS fait encore augmenter l'impact du sel, cette fois de 30 à $50 \%$ (Fig. 4). Par contre ces effets physiologiques de niveaux élevés $\left(\mathrm{n}_{5}\right)$ apparaissent inexistants chez l'acacia (Fig. 4), ce qui indiquerait que cet arbre est plus résistant que l'eucalyptus à ce type de polluants. Un résultat similaire [8] a montré que Pittosporum tobira est plus résistante que Pinus pinea, Quercus ilex et Acer opalus exposés à différents traitements $(\mathrm{NaCl} 30 \mathrm{~g} / \mathrm{L}$ et/ou LAS $250 \mathrm{mg} / \mathrm{L})$. Nous constatons également au cours de ces expériences, qu'une aspersion par l'eau de mer polluée (EMP) engendre des nécroses foliaires sur les houppiers, aussi bien pour l'acacia que pour l'eucalyptus, avec $30 \%$ de feuilles fortement nécrosées de niveau $\mathrm{n}_{5}$ chez l'eucalyptus et seulement $13 \%$ chez l'acacia (Fig. 4). Il apparaît donc que les embruns marins provenant d'une eau de mer polluées in situ (EMP) entraînent sur les feuilles d'eucalyptus des effets tout à fait similaires à ceux du traitement EMS + LAS + HC avec $80 \%$ de feuilles nécrosées $\left(\mathrm{n}_{3}+\mathrm{n}_{4}+\mathrm{n}_{5}\right)$ pour le traitement EMP contre $84 \%$ pour le traitement EMS + LAS + HC. La même conclusion a été trouvée chez acacia avec $70 \%$ de feuilles nécrosées pour ces deux traitements (Fig. 4)

\subsubsection{Dosage des LAS sur les feuilles}

Après les expériences de simulation, on relève des concentrations en LAS de 86,2 $\pm 0,1 \mu \mathrm{g} / \mathrm{kg}$ de MF sur les feuilles d'A. cyanophylla et de $89,5 \pm 0,1 \mu \mathrm{g} / \mathrm{kg}$ de MF sur les feuilles d' $E$. gomphocephala (Tab. III). Remarquons que cette concentration de tensioactifs anioniques présente sur les feuilles d' $E$. gomphocephala est environ 6 fois plus importante que celle trouvée sur la même espèce in situ.

\subsubsection{Mouillabilité des surfaces foliaires}

Nous constatons que les tensioactifs anioniques en support salin (traitement EMS+LAS) affecte fortement la mouillabilité des feuilles en diminuant l'angle de contact des gouttes d'eau sur les feuilles d'A. cyanophylla de $45 \%$ et sur les feuilles d'E. gomphocephala de $40 \%$ par rapport au témoin (Fig. 5). Les traitements EMS + LAS + HC et EMP ont un impact encore plus important sur les valeurs de cet angle de contact. En effet celles-ci 
Niveau de dommage (S)

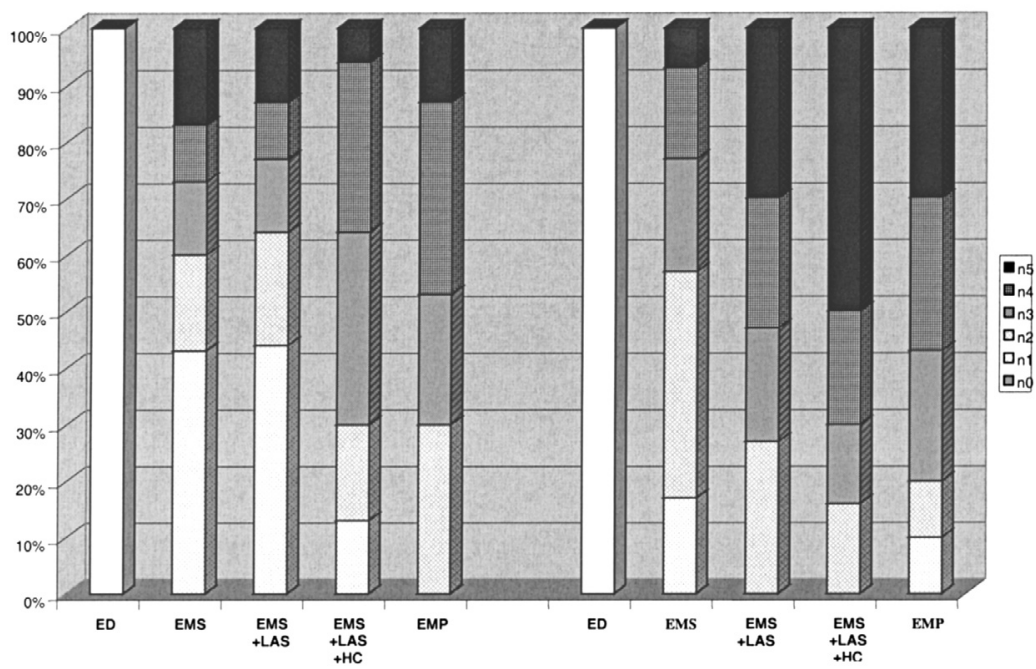

Acacia cyanophylla

Eucalyptus gomphocephala
Figure 4. Pourcentages des différents niveaux de dommage (S) sur les feuilles d'Acacia cyanophylla et d'Eucalyptus gomphocephala en fonction des différents traitements. $(\mathrm{ED}=$ eau distillée $; \mathrm{EMS}=$ eau de mer synthétique $(38 \mathrm{~g} / \mathrm{L}) ; \mathrm{EMS}+\mathrm{LAS}=\mathrm{EMS}$ + tensioactifs anioniques $50 \mu \mathrm{g} / \mathrm{L}$; EMS + LAS + $\mathrm{HC}=\mathrm{EMS}+\mathrm{LAS}+$ hydrocarbures $1,25 \mathrm{~mL} / \mathrm{L}$; $\mathrm{EMP}=$ eau de mer polluée) .

Angle de contact $\left(\theta^{\circ}\right)$

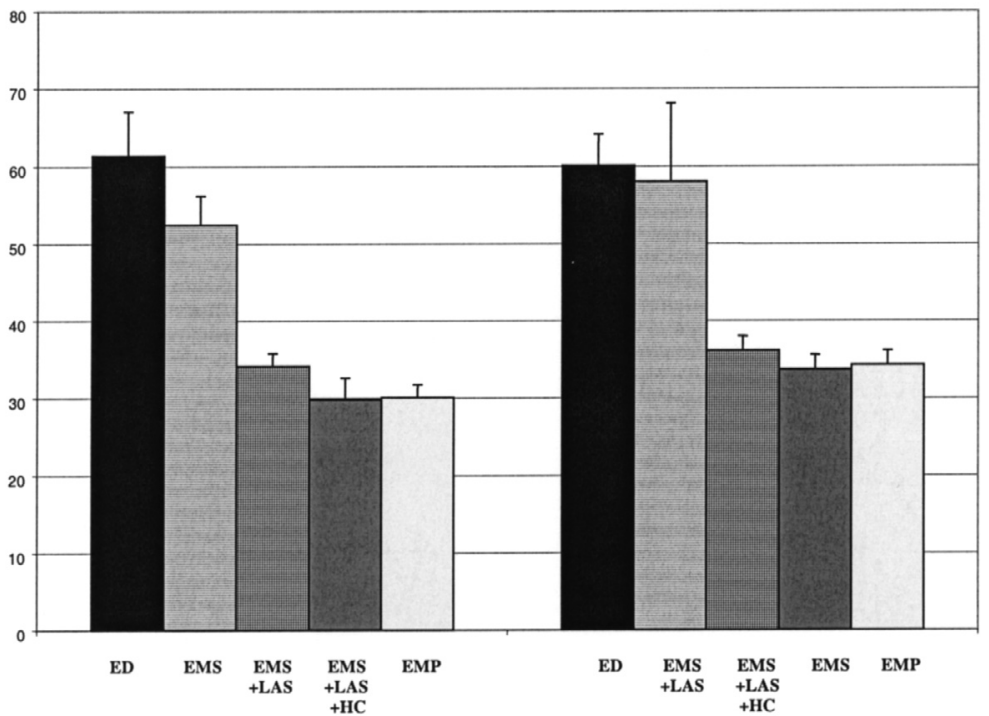

Acacia cyanophylla

Eucalyptus gomphocephala

Figure 5. Variation de l'angle de contact des gouttes d'eau $\left(\theta^{\circ}\right)$ sur les feuilles d'Acacia cyanophylla et d'Eucalyptus gomphocephala en fonction des différents traitements $(\mathrm{ED}=$ eau distillée $; \mathrm{EMS}=$ eau de mer synthétique $(38 \mathrm{~g} / \mathrm{L})$; EMS + LAS = $\mathrm{EMS}+$ tensioactifs anioniques $50 \mu \mathrm{g} / \mathrm{L}$; EMS + $\mathrm{LAS}+\mathrm{HC}=\mathrm{EMS}+\mathrm{LAS}+$ hydrocarbures $1,25 \mathrm{~mL} / \mathrm{L} ; \mathrm{EMP}=$ eau de mer polluée). $(n=40)$. Les barres verticales correspondent à l'écart type.

Tableau III. Concentrations des LAS (exprimée en $\mu \mathrm{g} / \mathrm{kg}$ de MF) présents sur les feuilles d'Acacia et l'Eucalyptus en pépinière.

\begin{tabular}{lccccccccccc}
\hline $\mathbf{N}^{\circ}$ des mesures & 1 & 2 & 3 & 4 & 5 & 6 & 7 & 8 & 9 & 10 & Moy. \\
\hline Acacia & 94,0 & 89,9 & 78,4 & 97,7 & 86,0 & 78,3 & 86,4 & 79,2 & 92,5 & 79,9 & 86,2 \\
Eucalyptus & 92,6 & 89,5 & 89,2 & 89,0 & 98,9 & 90,0 & 77,6 & 86,8 & 89,5 & 92,2 & 89,5 \\
\hline
\end{tabular}

diminuent alors l'angle $\theta^{\circ}$ de $51 \%$ chez l'acacia et de $44 \%$ chez l'eucalyptus (Fig. 5).

\subsubsection{Observation des surfaces foliaires au microscope électronique à balayage}

Les feuilles des plants témoins fumigés par de l'eau distillée d'A. cyanophylla (face adaxiale) apparaissent au MEB recou- vertes d'une couche amorphe et épaisse de cires épicuticulaires sans aucune altération (Fig. 6). Leurs stomates, uniquement présents sur la face abaxiale, sont enfoncés dans l'épiderme et montrent à l'extérieur une chambre protégée par des cires épistomatiques (Fig. 7). De son côté, la face adaxiale de feuilles d'E. gomphocephala témoins montre une couche des cires épicuticulaires continue avec des microstructures cristallines rondes 
Tableau IV. Détermination de l'état d'endommagement foliaire suivant les critères de Sauter et Voss (S.V.).

\begin{tabular}{lccccc}
\hline $\mathbf{N}^{\circ}$ figure & $\mathbf{6}$ & $\mathbf{7}$ & $\mathbf{8}$ & $\mathbf{9}$ & $\mathbf{1 0}$ \\
\hline Espèce/face & Acacia/adax. & Acacia/abax. & Eucaly./adax. & Eucaly./abax. & Eucaly./abax. \\
Traitement & ED & EMS + LAS + HC & ED & EMS & EMS + LAS + HC \\
Critère S.V. & I & III & I & II & IV \\
\hline
\end{tabular}

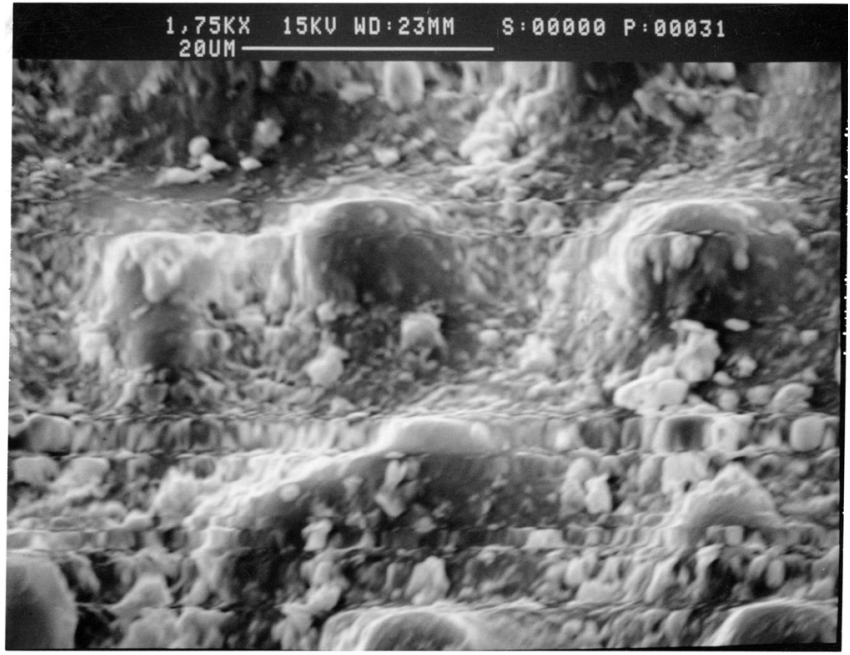

Figure 6. Aspects des cires épicuticulaires sur la face adaxiale d'une jeune feuille de 6 mois d'Acacia cyanophylla témoin, fumigé par l'eau distillée $(\times 1750)$.

(Fig. 8). Nous mettons en évidence que les différents traitements appliqués (EMS ; EMS + LAS et EMS + LAS + HC) engendrent un véritable « décirage » des surfaces foliaires avec une agrégation des cires épicuticulaires restantes (Figs. 9 et 10) avec l'apparition sur les feuilles de nécroses de différents degrés. Sur chaque surface foliaire traitée les dommages au niveau des cires ont été classés selon les critères de Sauter et Voss. Nous constatons (Tab. IV ; Figs. 6 et 7) que l'ensemble des composés polluants : tensioactifs+hydrocarbures en support salin (EMS + LAS + HC) provoque une sévère exfoliation de la couche cireuse épicuticulaire ainsi que des cires épistomatiques sur les feuilles d'A. cyanophylla avec un passage de l'état I à l'état III. Ce même traitement sur E. gomphocephala (Figs. 8 et 10) provoque une dégradation complète de la couche des cires épicuticulaires et épistomatiques avec un passage de l'état I à l'état IV.

\section{DISCUSSION ET CONCLUSIONS}

Nos analyses des LAS dans l'eau de mer (10,8 $\mu \mathrm{g} / \mathrm{L})$ et sur les feuilles des arbres côtiers du Cap Bon montrent pour la première fois la forte présence de ces composés polluants dans cette région où des dépérissements d'arbres sont observables. Par comparaison, des quantités de tensioactifs anioniques de 0 à $11,8 \mu \mathrm{g} / \mathrm{L}$ ont été détectées dans l'eau de mer de Porquerolles en France [35] où des dépérissements du même type sont également présents. De même en Italie, il a été déterminé des quantités importantes des tensioactifs anioniques non biodégradables

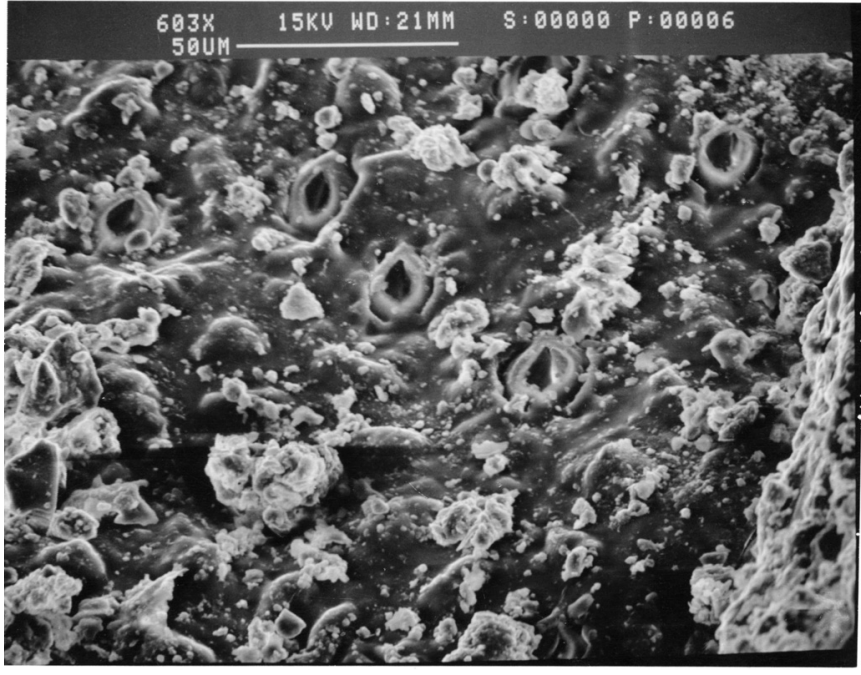

Figure 7. Aspects des cires épicuticulaires sur la face abaxiale d'une jeune feuille de 6 mois d'Acacia cyanophylla après traitement par l'eau de mer synthétique + tensioactifs anioniques + hydrocarbures $(\times 603)$.

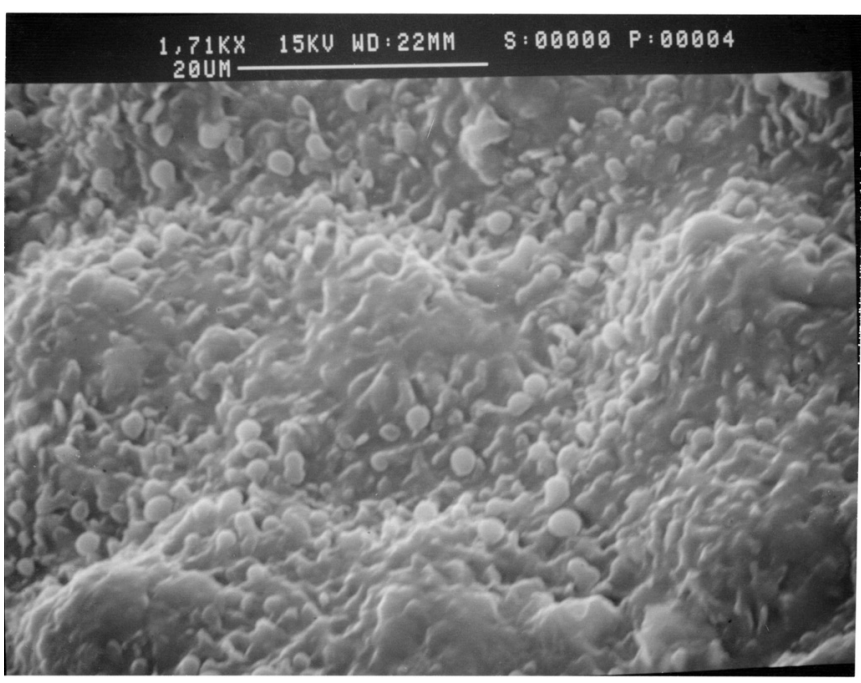

Figure 8. Aspects de la face adaxiale d'une jeune feuille de 6 mois d'Eucalyptus gomphocephala témoin, fumigé par l'eau distillée $(\times 1710)$

dans la mer en face du parc de San Rossore où de nombreuses Pinèdes dépérissantes existent : jusqu'à $37 \mathrm{mg} / \mathrm{L}$ exprimés comme MBAS (Substances Actives au Bleu de Méthylène) [19]. 


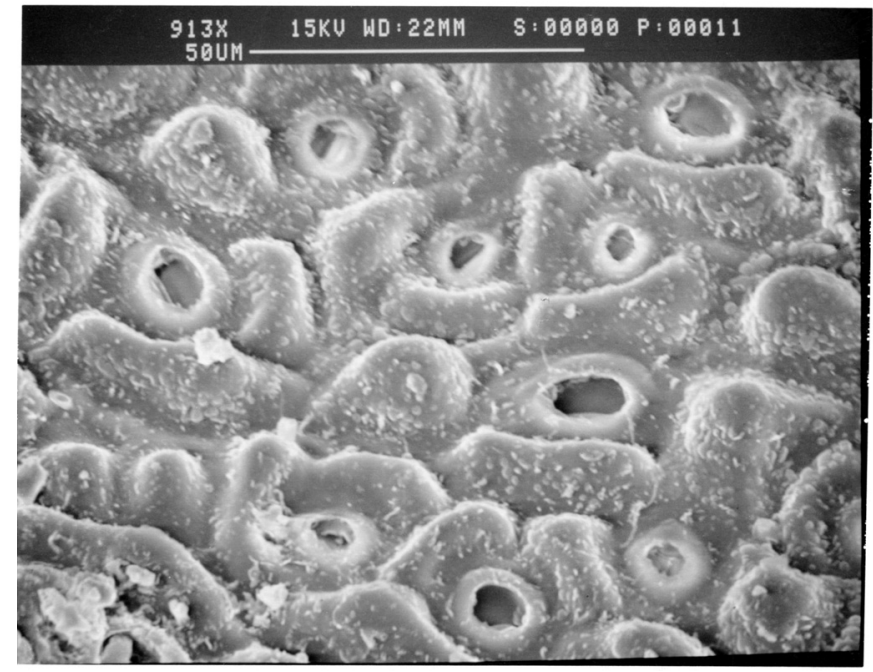

Figure 9. Aspects de la face abaxiale d'une jeune feuille de 6 mois d'Eucalyptus gomphocephala après traitement par l'eau de mer synthétique $(\times 913)$.

Au niveau du statut hydrique des arbres du Cap Bon, nous constatons qu'exposés aux embruns marins pollués, les feuilles d'A. cyanophylla et d'E. gomphocephala présentent une réduction marquée de leur teneur en eau relative (29\% chez l'eucalyptus). Une diminution similaire de $20 \%$ de RWC a été mesurée sur Pistacia atlantica sous l'effet des embruns marins pollués par les tensioactifs et les hydrocarbures et de $24 \%$ sur les feuilles de Ficus benjamina dans la région de Barcelone [29]. Cette diminution de RWC, qui correspond à des pertes d'eau foliaire, explique l'altération du statut hydrique des feuilles régulièrement signalée chez les arbres soumis aux embruns marins pollués $[4,6]$.

Au niveau du statut minéral des arbres du Cap Bon, on note une augmentation des teneurs en $\mathrm{Na}^{+}$dans les tissus foliaires d'A. cyanophylla et d'E. gomphocephala et ceci d'autant plus que l'on se rapproche du rivage (augmentation de $\mathrm{Na}^{+}$de 0,54 à $1,62 \%$ chez l'acacia et de 0,39 à $2,3 \%$ chez l'eucalyptus). De fortes accumulations d'ions $\mathrm{Na}^{+}$et $\mathrm{Cl}^{-}$foliaires avaient déjà été observées chez les Araucaria heterophylla du littoral d'Australie exposés aux embruns marins pollués par les tensioactifs anioniques (LAS) [11]. Parallèlement, nous avons dans les mêmes conditions une baisse des teneurs en $\mathrm{K}^{+}$(de 0,47 à $0,33 \%$ chez l'acacia et de 0,72 à $0,22 \%$ chez l'eucalyptus) ainsi que du rapport $\mathrm{K} / \mathrm{Na}$. Des études antérieures avaient déjà montré chez Picea abies, des teneurs en $\mathrm{K}^{+}$des aiguilles de sujets dépérissants $(0,40 \%)$ inférieures à celles de sujets sains $(0,55 \%)$ [5] et un rapport K/Na en baisse chez Pinus pinea et Quercus ilex traités par des solutions salines riches en LAS (de 0 à 240 ppm) [24]. Il est vraisemblable que l'entrée massive des ions $\mathrm{Na}^{+}$dans les feuilles, ions en compétition au niveau cellulaire avec les ions $\mathrm{K}^{+}$, entraîne une excrétion de ces derniers pour maintenir l'équilibre des charges dans la cellule [3].

Les expériences de simulation en laboratoire montrent clairement de leurs côtés, qu'il est possible de reproduire lors de courtes expositions, les symptômes observés sur le terrain, ceci en soumettant la végétation à des mélanges de $\mathrm{NaCl}$, de ten-

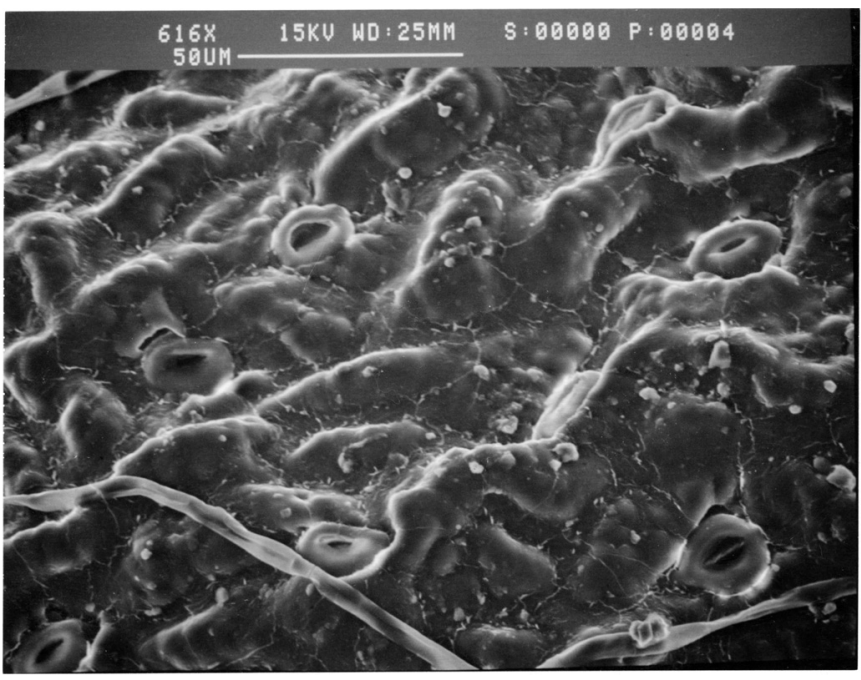

Figure 10. Aspects de la face abaxiale d'une jeune feuille de 6 mois d'Eucalyptus gomphocephala après traitement par l'eau de mer synthétique + tensioactifs anioniques + hydrocarbures $(\times 616)$.

sioactifs anioniques comme les Linear alkylbenzène sulfonates et/ou à des hydrocarbures de type fuel. Elles confirment que l'ensemble $\mathrm{NaCl}+\mathrm{LAS}$ a un rôle déterminant dans l'apparition des dommages des feuilles d'A. cyanophylla et d'E. gomphocephala. Un résultat similaire avait déjà été obtenu sur les pins d'Alep lors de fumigations en laboratoire [31]. Nous constatons que si le $\mathrm{NaCl}$ appliqué seul est capable de causer des nécroses foliaires (traitement EMS), leur pourcentage est toutefois très nettement inférieur à celui d'un traitement à l'eau de mer polluée (EMP) ou à un traitement $\mathrm{NaCl}+\mathrm{LAS}(\mathrm{EMS}+$ LAS). Mais surtout nos résultats montrent pour la première fois, le rôle non négligeable des hydrocarbures dans l'apparition des dommages foliaires. Lorsque les LAS et les HC arrivent au contact de la surface des feuilles, les observations en microscope électronique à balayage mettent alors en évidence de profonds changements de la morphologie des cires épicuticulaires chez les deux espèces étudiées. Cependant, le MEB ne nous renseigne pas sur les possibles modifications au niveau des cires intracuticulaires. L'effet cumulé des LAS plus des hydrocarbures en support salin (EMS + LAS + HC) apparaît donc comme le plus perturbateur pour la surface foliaire et pour la physiologie de la feuille. De nombreux auteurs ont décrit une modification de la structure des cires cuticulaires sous l'action des tensioactifs de type LAS chez les végétaux ligneux [21, 22, 27]. De même une dégradation par des LAS marqués [ $\left.{ }^{35} \mathrm{~S}\right]$ des cires épicuticulaires sur les aiguilles de Pinus halepensis a été trouvée 2 fois supérieure en solution d'eau de mer comparativement à celle obtenue en solution d'eau distillée [16, 32]. D'autres résultats ont montré l'agrégation des microtubules de cires de la chambre épistomatique [2, 7, 23], et indiqué que les tensioactifs seuls provoquent une remarquable exfoliation de la couche cireuse épicuticulaire [19] avec alors l'apparition sur des aiguilles de Pinus pinaster traitées avec $\mathrm{NaCl}$ et des tensioactifs, de lésions semblables à celles de plantes naturellement exposées à l'aérosol marin pollué. Il a été aussi signalé [23] que la pulvérisation des détergents anioniques (solution à 30 ppm 
avec plus de 10 pulvérisations) favorise une coalescence des fibres cireuses et la formation d'un bouchon qui en disparaissant découvre la chambre stomatique. Cela est du selon ces auteurs, à une action phytotoxique directe des tensioactifs anioniques sur les cires. D'autres auteurs [21] ont montré que l'action synergique entre les tensioactifs anioniques et le chlorure de sodium était la cause principale de la destruction des cires épicuticulaires et en particulier de la couche de protection des stomates.

Nos études de terrain comme nos études au laboratoire montrent clairement que la présence de polluants comme les LAS entraîne une diminution de l'angle de contact des gouttes d'eau sur les feuilles, c'est à dire une augmentation de la mouillabilité des surfaces foliaires. Chez A. cyanophylla comme chez E. gomphocephala la mesure de l'angle de contact des gouttes d'eau, montre que c'est l'effet conjugué de deux polluants en support salin (traitement $\mathrm{EMS}+\mathrm{LAS}+\mathrm{HC}$ ) qui affecte le plus la mouillabilité des feuilles, ce qui confirme bien nos observations parallèles au microscope électronique à balayage sur la dégradation des cires épicuticulaires. Ces tensioactifs présents dans les aérosols marins, réduisent la tension de surface des gouttes et entraînent un plus grand étalement de celles-ci, ce qui va par conséquence amener un plus grand étalement des polluants sur la surface de la feuille et favoriser par la suite leur pénétration [30].

Toutes ces recherches effectuées dans la région du Cap Bon montrent une forte similitude des perturbations physiologiques des arbres dépérissants de Tunisie et de ceux observés dans le sud de l'Europe. Parallèlement les expériences au laboratoire suggèrent fortement que les causes de tous ces phénomènes de dépérissement sont communes et liées à la présence anormale de tensioactifs dans les embruns marins. À partir de nos différents résultats et de travaux précédents [18, 29, 31], nous émettons l'hypothèse que les mécanismes suivants se déroulent pour expliquer ces dépérissements : les tensioactifs anioniques présents dans l'eau de mer, mais également les hydrocarbures, après un large étalement des embruns sur les feuilles, dégradent dans un premier temps la barrière lipidique constituée par les cires cuticulaires. Mais on ne sait toujours pas : d'une part si seules les cires épicuticulaires sont touchées ou si les cires intracuticulaires sont également atteintes ; d'autre part si cette dégradation des cires résulte d'un effet directe par contact avec l'eau de mer polluée, d'un effet indirect via des perturbations de leur métabolisme ou des deux. Il est aussi important de tenir compte du fait que les tensioactifs, suite à leurs interactions avec la cuticule selon des mécanismes encore pas complètement élucidés, favorisent la pénétration des ions minéraux [9]. $\mathrm{Na}^{+}, \mathrm{Cl}^{-}$, mais aussi les différents polluants, pourraient alors pénétrer en grandes quantités dans les tissus foliaires via la cuticule où ils vont exercer directement ou indirectement leurs effets néfastes sur le métabolisme cellulaire. Par contre nous pensons que la tension superficielle de ces embruns pollués et le caractère hydrophobe de la cuticule bordant l'ostiole ne permettent pas le passage direct de ceux-ci à travers les stomates $[13,15]$. Cependant certains auteurs indiquent que l'eau de mer polluée pourrait passer au travers des stomates, surtout suite aux fortes perturbations physiologiques de ceux-ci entraînées par les tensioactifs [7].

Si la toxicité du $\mathrm{NaCl}$ est selon notre avis et celui d'autres auteurs [22, 24, 28] la cause majeure du dépérissement des plantes du littoral (essentiellement $\mathrm{Na}^{+}$), des travaux donnent, en plus du $\mathrm{Na}^{+} \mathrm{Cl}^{-}$, un rôle perturbateur significatif aux tensioactifs et aux hydrocarbures qui ont pénétré conjointement dans les tissus foliaires. En effet ces produits en solubilisant entre autre les parois cellulaires, auraient une action directe sur les organites cellulaires, principalement les chloroplastes, et expliqueraient la réduction de l'activité photosynthétique constatée chez les arbres dépérissants [7, 25,26]. Rappelons que les hydrocarbures agissent à des concentrations 10 fois plus élevées que les détergents, mais ils sont 10 fois plus abondants (en région méditerranéenne, les détergents sont estimés à une concentration de $0,1 \%$ et le fioul à $1 \%$ dans l'eau de mer) [38].

Parallèlement à ces désordres physiologiques, l'altération des cires va également faciliter le départ de l'eau contenue dans les tissus foliaires, déficit hydrique amplifié par l'altération du fonctionnement des stomates [7, 18] et par l'arrivée massive de $\mathrm{Na}^{+} \mathrm{Cl}^{-}$dans ces tissus et le déséquilibre osmotique (diminution du potentiel hydrique) qui en résulte.

Remerciements : Ce travail a bénéficié du soutien financier du Secrétariat d'État à la Recherche Scientifique et à la Technologie en Tunisie.

\section{RÉFÉRENCES}

[1] Albouchi A., Sebeï H., Mezni M.Y., El Aouni M.H., Influence de la durée d'acclimatation sur l'endurcissement à la sécheresse d'Acacia cyanophylla Lindl., Ann. For. Sci. 58 (2001) 519-528.

[2] Altieri A., Del caldo L., Manes F., Morphology of epicuticular waxes in Pinus pinea needles in relation to season and pollution climate, Eur. J. For. Path. 24 (1994) 79-91.

[3] Amtmann A., Sanders D., Mechanisms of $\mathrm{Na}^{+}$uptake by plant cells, Adv. Bot. Res. 29 (1999) 75-112.

[4] Badot P.M., Badot M.J., Dépérissement du pin d'Alep sous l'effet des embruns marins pollués : symptômes macroscopiques et mise en évidence des perturbations hydriques dans les aiguilles, Annales Scientifiques de l'Univ. de Franche Comté 3 (1991-1995) 37-43.

[5] Badot P.M., Garrec J.P., Millet B., Badot M.J., Mercier J., Dépérissement et état hydrique des aiguilles chez le Picea abies, Can. J. Bot. 66 (1988) 1693-1701.

[6] Badot P.M., Richard B., Lucot E., Badot M.J., Garrec J.P., Water disturbances and needle surface alterations in Pinus halepensis Mill. Trees exposed to polluted sea-spray, in: Ecotoxicology of an compartment, Société d'Écotoxicologie Fondamentale et Appliquée, Rouen, 1996, pp. 179-189.

[7] Bussotti F., Botacci A., Grossoni P., Mori B., Tani C., Cytological and structural changes on Pinus pinea L. needles following the application of an anionic surfactant, Plant Cell Environ. 20 (1997) 513520 .

[8] Bussotti F., Grossoni P., Pantani F., The role of marine salt and surfactants in the decline of Thyrrhenian coastal vegetation in Italy, Ann. Sci. For. 52 (1995) 251-261.

[9] Chamel A., Foliar absorption of herbicides: study of the cuticular penetration using isolated cuticules. Physiol. Vég. 4 (1986) 24.

[10] Devèze L., Sigoillot J.C., Les arbres malades de la mer, Eau Aménagement 19 (1978) 13-24.

[11] Dowden H.G.M., Lambert M.J., Environmental factors associated with a disorder affecting tree species on the coast of new south wales with particular reference to Norfolk island Pines Araucaria heterophylla, Environ. Pollut. 19 (1979) 71-84.

[12] El Ayeb N., Dépérissement forestier au littoral par les embruns marins pollués, Thèse de Doctorat, Univ. Tunis El Manar, 2001, $137 \mathrm{p}$. 
[13] Garrec J.P., Barrois A., Caractéristiques de la fixation et de la pénétration corticale. Passage du phosphite dipotassique et de l'eau au travers d'écorces isolées, Environ. Exp. Bot. 32 (1992) 11-23.

[14] Garrec J.P., El Ayeb N., Il problema degli aerosol marini inquinati in Francia e Tunisia. Danni alla vegetazione costiera ed inquinamento da tensioattivi, Linea Ecologica 33 (2001) 51-54.

[15] Garrec J.P., Laitat E., Richardin I., Rose C., Dépérissement forestier et perturbations minérales aux niveaux histologique et cellulaire dans les aiguilles de Picea abies L. Étude par microanalyse X, Ann. Sci. For. 48 (1991) 321-332.

[16] Garrec J.P., Richard B., Badot P.M., The impact of polluted seaspray on the needle surface of Pinus halepensis, in: EUROSILVA Workshop, 1994.

[17] Garrec J.P., Sigoillot J.C., Les arbres malades de la mer, La Recherche 245 (1992) 940-941.

[18] Gellini R., Pantani F., Grossoni P., Colloque International d'Écologie littorale méditerranéenne, Société d'Écologie, 1986, 42 p.

[19] Gellini R., Pantani F., Grossoni P., Bussotti F., L'influence de la pollution marine sur la végétation côtière italienne, Bull. Ecol. 18 (1987) 213-219.

[20] Gellini R., Pantani F., Grossoni P., Bussotti F., Barbolani E., Rinallo C., Survey of the deterioration of the coastal vegetation in the park of San Rossore in central Italy, Sonderdruck aus Eur. J. For. Pathol. 13, 5-6 S (1983) 296-304.

[21] Gellini R., Pantani F., Grossoni P., Bussotti F., Barbolani E., Rinallo C., Further investigation on the causes of disorder of the coastal vegetation in the park of San Rossore (Central Italy), Sonderdruck aus Eur. J. For. Pathol. 15, 3 S (1985) 145-157.

[22] Grieve A.M., Pitman M.G., Salinity damage to Norfolk Island pines caused by surfactants. III. Evidence for stomatal penetration as the pathway of salt entry to leaves, Aust. J. Plant. Physiol. 5 (1978) 397 413.

[23] Guidi L., Lorenzini G., Soldatini G.F., Ricerche sulla fitotossicità degli aerosol marini in condizioni simulate, con particolare riferimento al microinquinamento da tensioattivi, Estratto da Agricoltura Ricerca, Istituto di Tecnica e Propaganda Agraria, Roma 71 (1986) 45-48.

[24] Guidi L., Lorenzini G., Soldatini G.F., Phytotoxicity of sea-water aerosols on forest plants with special reference to the role of surfactants, Environ. Exp. Bot. 28 (1988) 85-94.

[25] Innamorati M., Effeti dell'aerosol da acque marine con tensioattivi su Pinus pinea, Relaz. Contr C.E.E. C.N.R., ENW, 750 I, 1986 (EDB).
[26] Itoh H., Izawa S., Shibata K., Disintegration of chloroplasts with dodecylbenzenesulfonate as mesured by flattening effect and size distribution, Biochim. Biophys. Acta 69 (1963) 147-151.

[27] Keever J.G.J., Jacobson J.S., Response of Glycine max (L.) Merrill. To simulated acid rain. Environmental and morphological influences on the foliar leaching of ${ }^{86} \mathrm{Rb}$, Fields Crops Res. 6 (1983) 241-250.

[28] Loglio G., Degli Innocenti N., Gellini R., Pantani F., Cini R., Detergents as a condition of pollution from coastal marine aerosol, Mar. Pollut. Bull. 20 (1989) 115-119.

[29] Maru J., Savé R., Bayona J.M., Efectos de la contaminación química sobre la vegetación del frente litoral de Barcelona, Retema Nov.Déc. (1997) 25-34.

[30] Meli C., Resch F., Embruns marins pollués (Synthèse bibliographique), Labo. Océanographie physique de Toulon, Univ. Toulon, 1990, $32 \mathrm{p}$.

[31] Richard B., Étude des perturbations structurales et chimiques des cires cuticulaires des aiguilles de Pinus halepensis Mill. dépérissants en relation avec l'exposition aux embruns marins pollués: implication d'un tensioactif, le dodecylbenzene sulfonate linéaire, Thèse de Doctorat, Univ. Henri Poincaré Nancy I, 1996, 107 p.

[32] Richard B., Grieu P., Badot P.M., Garrec J.P., Influence of marine salts on the localization and accumulation of surfactant in the needles of Pinus halepensis Mill, Ann. Sci. For. 53 (1996) 921-930.

[33] Sauter J.J., Voss J.U., SEM-observations on the structural degradation of epistomatal waxes in Picea abies (L.) Karst. and its possible role in the "Fichtensterben", Eur. J. For. Path. 16 (1986) 408-423.

[34] Sigoillot J.C., Dispersion et dégradation bactérienne des tensioactifs dans les eaux littorales, Thèse de l'Université d'Aix-Marseille III, 1987, 206 p.

[35] Sigoillot J.C., Carriere F., Nguyen M.H., Pollution de la rade d'Hyères par les tensioactifs anioniques. Dispersion et potentialité de biodégradation du polluant, Sci. Rep. Port-Cros Natl. Park. Fr. 13 (1987) 85-95.

[36] Sigoillot J.C., Nguyen M.H., Devèze L., Pollution par les aérosols marins dans les îles d'Hyères, Trav. Sci. Parc Nation. Port-Cros Fr. 7 (1981) 45-54.

[37] Stammiti L., Le dépérissement de la végétation en bordure du littoral, in: Les éditions du S.T.U., Ministère de l'équipement, 1992, 131 p.

[38] Stammitti L., Garrec J.P., Arbres du littoral, arbres en péril ? Deux exemples de dépérissement des arbres en bordure du littoral français, Service Technique de l'Urbanisme, Courr. Environ. INRA 20 (1993) 49-58. 